\title{
VELOCIDAD SISTOLICA MAXIMA DE LA ARTERIA CEREBRAL MEDIA ELEVADA EN FETOS SIN ANEMIA: ¿FALSOS POSTIVOS?
}

\author{
Erasmo Huertas ${ }^{1, a}$, Ricardo Sugajara ${ }^{1, a}$, Walter Ventura ${ }^{1, a}$, Walter Castillo,a
}

\begin{abstract}
RESUMEN
La anemia fetal severa pude ser detectada intrautero a través de la medición de velocidad sistólica máxima de la Arteria Cerebral Media (VSmax-ACM). La tasa de falsos positivos de esta técnica de medición desafortunadamente es alta (34\%) por lo que se sugiere el correlato clínico materno fetal y descartar condiciones que eleven falsamente la VPS antes de tomar alguna decisión respecto del diagnóstico de ser de anemia fetal. Presentamos un caso de un feto con VSmax-ACM alterado por encima de 1.5 MoM que nació con valores de hemoglobina normales.
\end{abstract}

Palabras clave: Arteria cerebral media; Velocidad sistólica máxima; Anemia fetal (Fuente: DeCS BIREME).

\section{MAXIMUM SYSTOLIC SPEED OF HIGH AVERAGE CEREBRAL ARTERY IN FETUS WITHOUT ANEMIA: POSITIVE?}

\begin{abstract}
Severe fetal anemia can be detected in utero through the measurement of the maximum systolic velocity of the Middle Cerebral Artery (VSmax-MCA). The false positive rate of this measurement technique is unfortunately high (34\%), which is why the maternal-fetal clinical correlate is suggested and conditions that falsely elevate the SPV are ruled out before making any decision regarding the diagnosis of fetal anemia. We present a case of a fetus with altered VSmax-ACM above $1.5 \mathrm{MoM}$ who was born with normal hemoglobin values.
\end{abstract}

Keywords: Middle cerebral artery; Maximum systolic velocity; Fetal anemia (Source: MeSH NLM).

\section{INTRODUCCIÓN}

La Velocidad Sistólica Máxima de la Arteria Cerebral Media (VSmax-ACM) ha sido propuesta desde al año 2000 como el mejor método para detectar anemia fetal. Sin embargo, con el paso de los años se han publicado varios trabajos que reportan un número cada vez mayor de falsos positivos.

En el 2019 Martinez-Portilla publica una revisión sistemática y meta-análisis acerca del rendimiento del VSmax-ACM en la predicción de anemia fetal la cual concluye que en fetos no transfundidos la sensibilidad es de solo $73 \%$ para una tasa de falsos positivos de $10 \%$, lo cual quiere decir que uno de cada 4 fetos a los cuales se diagnostica anemia moderadasevera no estará afectado por esta patología. Presentamos el caso de un feto con VPS_ACM elevada con valores de hemoglobina normales al nacer debido a una cardiomiopatía secundaria a una malformación arteriovenosa cerebral que causa compromiso hemodinámico fetal.

\section{REPORTE DE CASO}

Tercigesta de 34 años, con antecedente de 2 partos vaginales, controlada 6 veces, es referida al INMP para neurosonografía fetal, por presentar megacisterna magna $(16 \mathrm{~mm})$ a las 31 semanas de gestación. La evaluación ecográfica en el Servicio de Medicina Fetal a las 33 semanas 1 día revela: "Cardiomegalia a predominio de cavidades derechas. Reflujo tricúspide. Leve derrame pericárdico y a nivel de SNC: Imagen anecoica elongada supratentorial occipital con flujo vascular turbulento de tipo venoso con ventriculomegalia leve. Polihidramnios moderado (ILA 31). La flujometría Doppler es reportada como: Aumento del IP de la arteria umbilical (1.47) asociado a aumento de la Velocidad Sistólica Máxima de la ACM $(89.72 \mathrm{~cm} / \mathrm{s})$ (Figura 1), Indice de Función Miocárdica Incrementado, Itsmo Aórtico Reverso (IFI 5) (Figura 2), IP del Ductus venoso por encima del percentil 95 (1.49) y Vena umbilical pulsátil , ". Concluye como Aneurisma de la Vena de Galeno con compromiso hemodinámico fetal por Anemia Severa. Se indica hospitalización para seguimiento fetal, maduración pulmonar y posibilidad de transfusión intrauterina. Dos días después se le realiza una prueba no estresante en la cual se evidencia dinámica uterina frecuente por lo que se indica tocólisis y una nueva ecografía la cual no muestra variación en los hallazgos Doppler respecto a la ecografía previa. La VSmax-ACM se encuentra en $99.72 \mathrm{~cm} / \mathrm{s}$. Es evaluada por neurología pediátrica quien concluye que el pronóstico del paciente es muy desfavorable.

Servicio de Medicina Fetal-Instituto Nacional Materno Perinatal. Lima, Perú.

Médico GínecoObstetra del Servicio de Medicina Fetal.

Citar como: Huertas E, Sugajara R, Ventura W, Castillo W. Velocidad sistolica maxima de la arteria cerebral media elevada en fetos sin anemia: ¿falsos postivos?.. Rev Peru Investig Matern Perinat 2020; 9(2): 42-46 DOI https://doi.org/10.33421/inmp.2020205

Recibido: 22-06-20 Aprobado: 30-06-20 

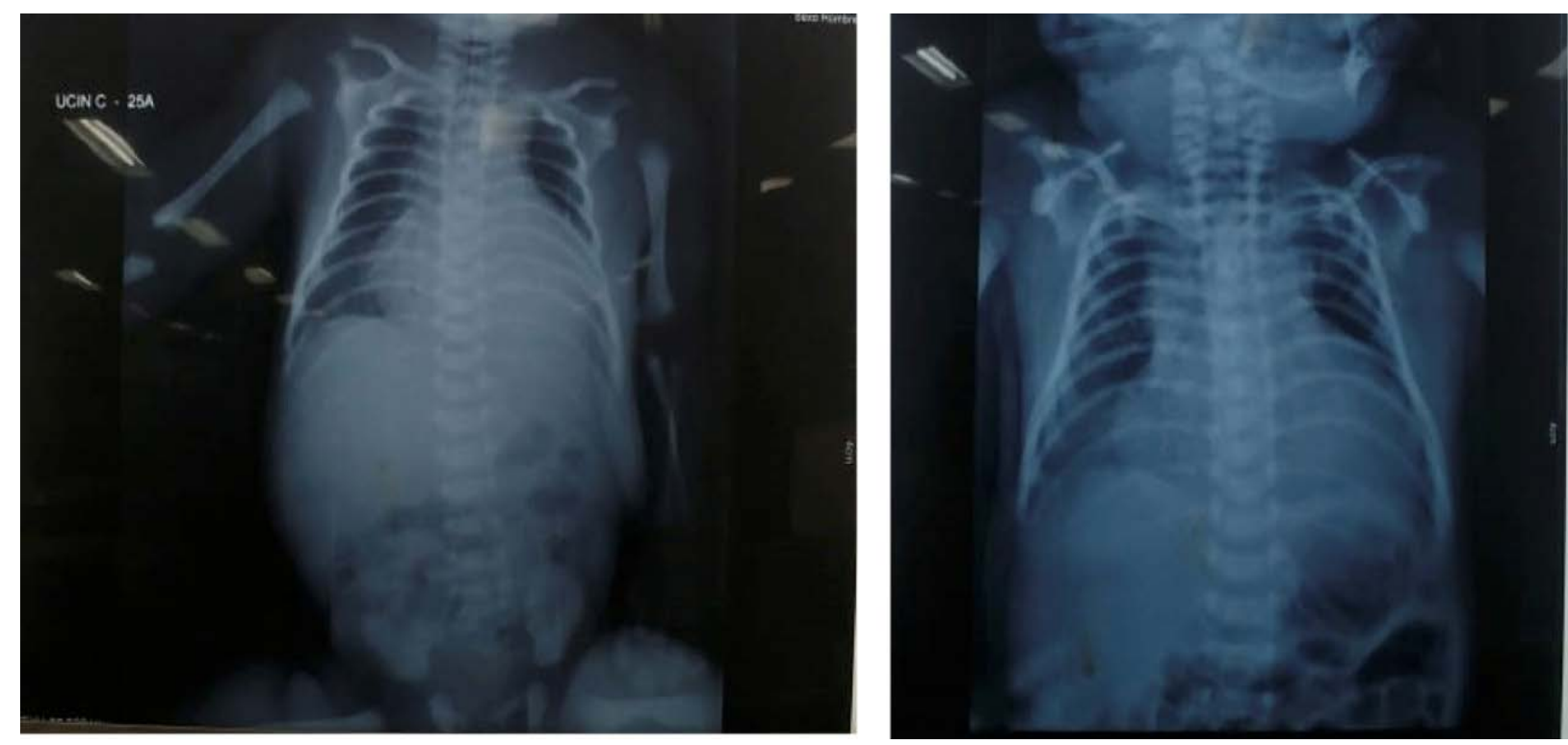

Figura 1

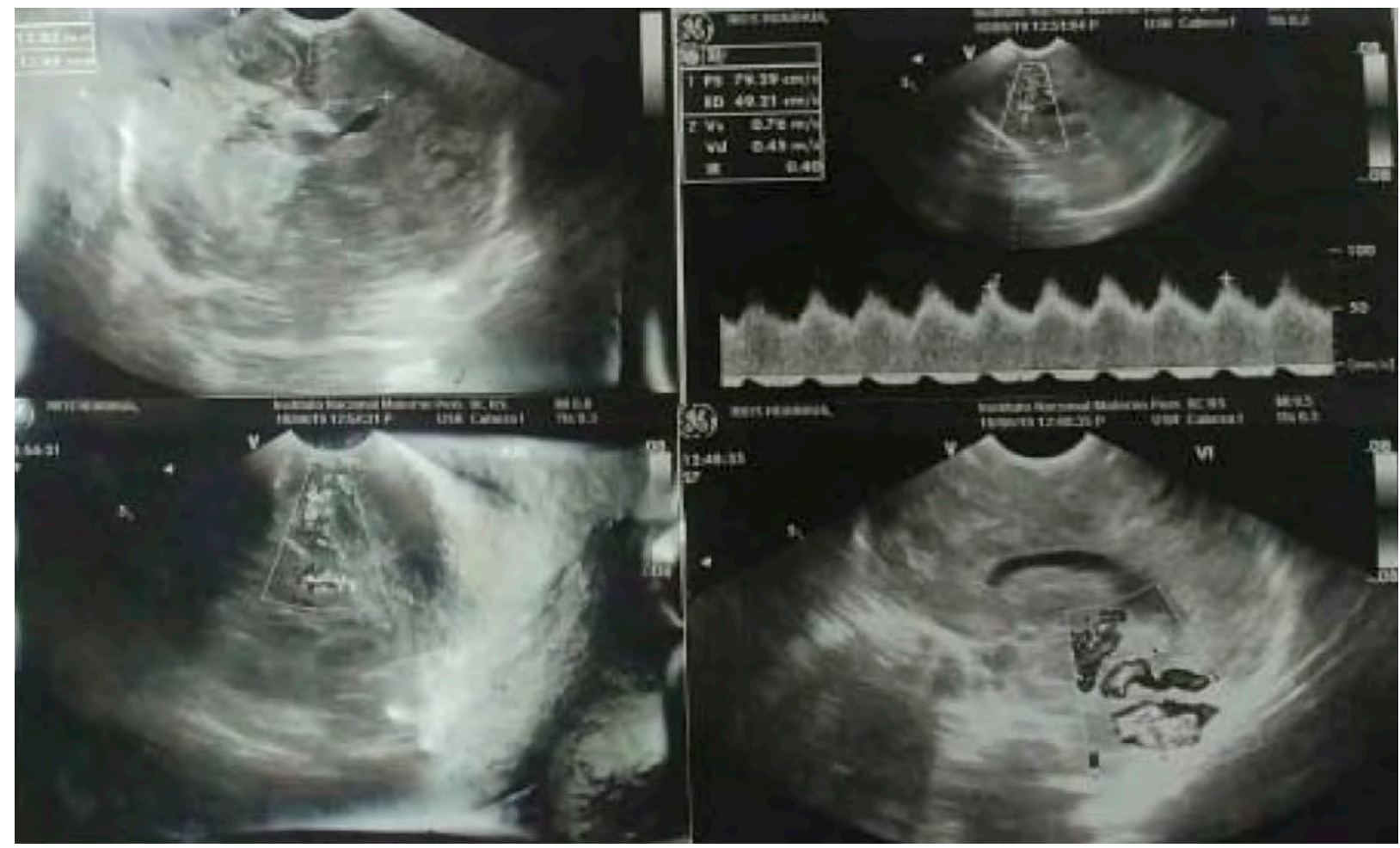

Figura 2

Una semana después de la primera evaluación en el servicio y luego de completar la maduración pulmonar con corticoides se programa cesárea electiva obteniéndose un recién nacido vivo sexo masculino, Peso: 2600gr, Talla: $48 \mathrm{~cm}$, Apgar: 6 y 8 . Tiene dificultad respiratoria por lo que se le coloca CPAP y se le hospitaliza. Se le solicita Hemoglobina y el resultado es $17.2 \mathrm{~g} / \mathrm{dL}$. (Hto: 52).
El examen neurológico del recién nacido es informado como: "Despierta con dificultad. Extensión de MS y semiflexion de $\mathrm{MI}$, con escasos movimientos de extremidades. Presenta hipotermia axial. Reflejo plantar presente pero débil, palmar no evaluable x VP. Moro no evaluable. Succión presente pero muerde y no es mantenida. ROT impresiona disminuido en MI. Cráneo: fontanela anterior palpable normotensa" (Figura 3). 


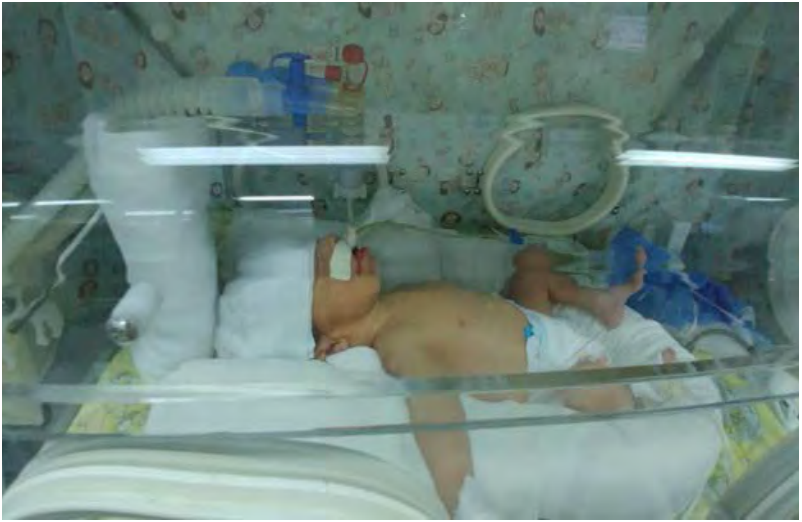

Figura 3
Se le solicita una Ecografía Transfontanelar, la cual se realiza ese mismo día y es informada como: "Hiperecogenicidad heterogénea en ambos hemisferios cerebrales. Espacio subaracnoideo amplio. Ventrículos discretamente dilatados. Se observó alteración en el flujo sanguíneo cerebral, observándose en fosa posterior imagen sugerente de aneurisma que también se observa en línea media. IR en ACA 0.40 con alteración en la regulación diastólica, se observa además shunt" (Figura 4).

Diagnóstico: Encefalopatía aguda. Sospecha de MAV: aneurisma de cerebral media.

PLAN: AngioTEM urgente. Monitoreo hemodinámico, hipertensión endocraneana (FC, patrón respiratorio y

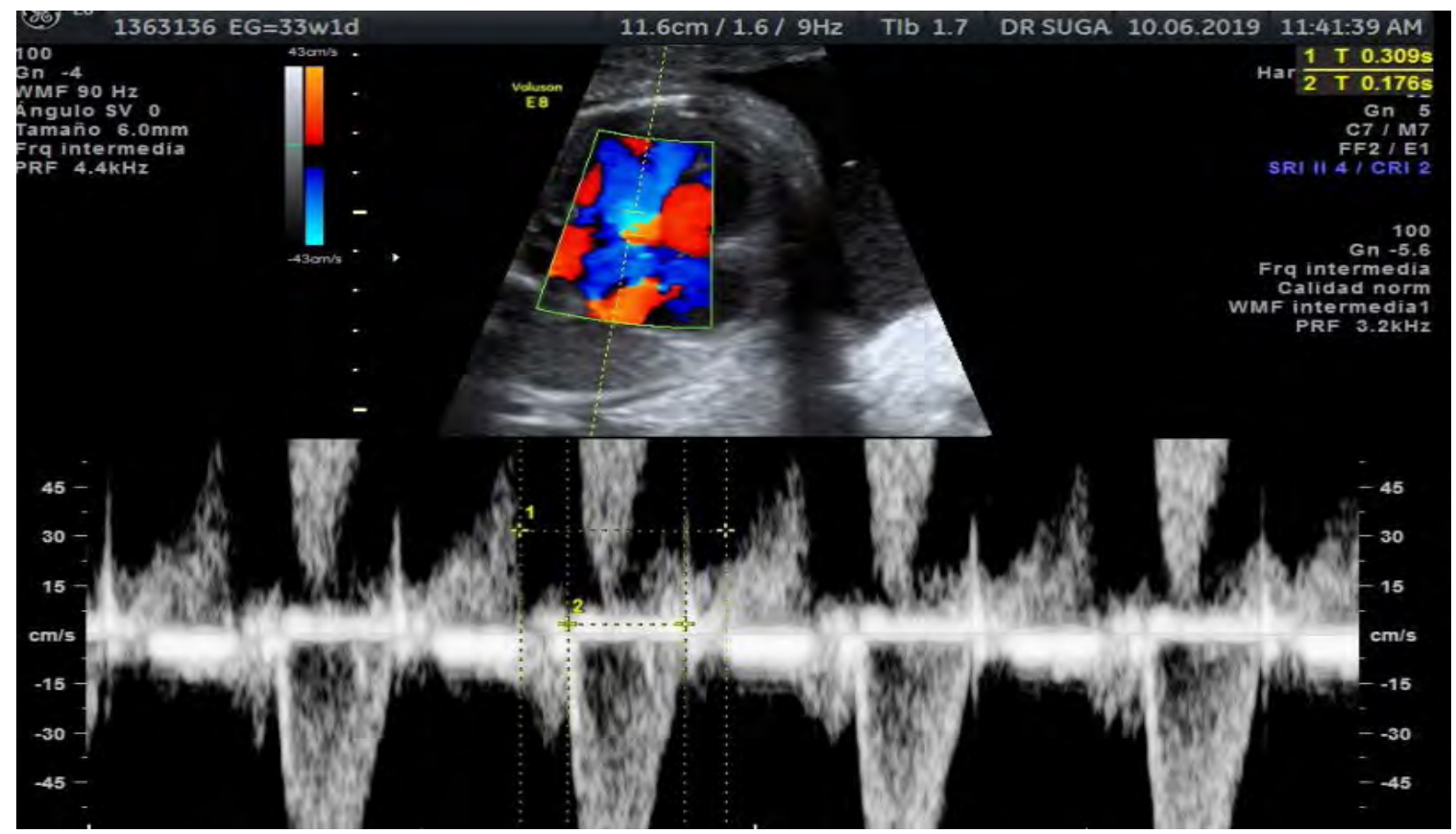

Figura 4
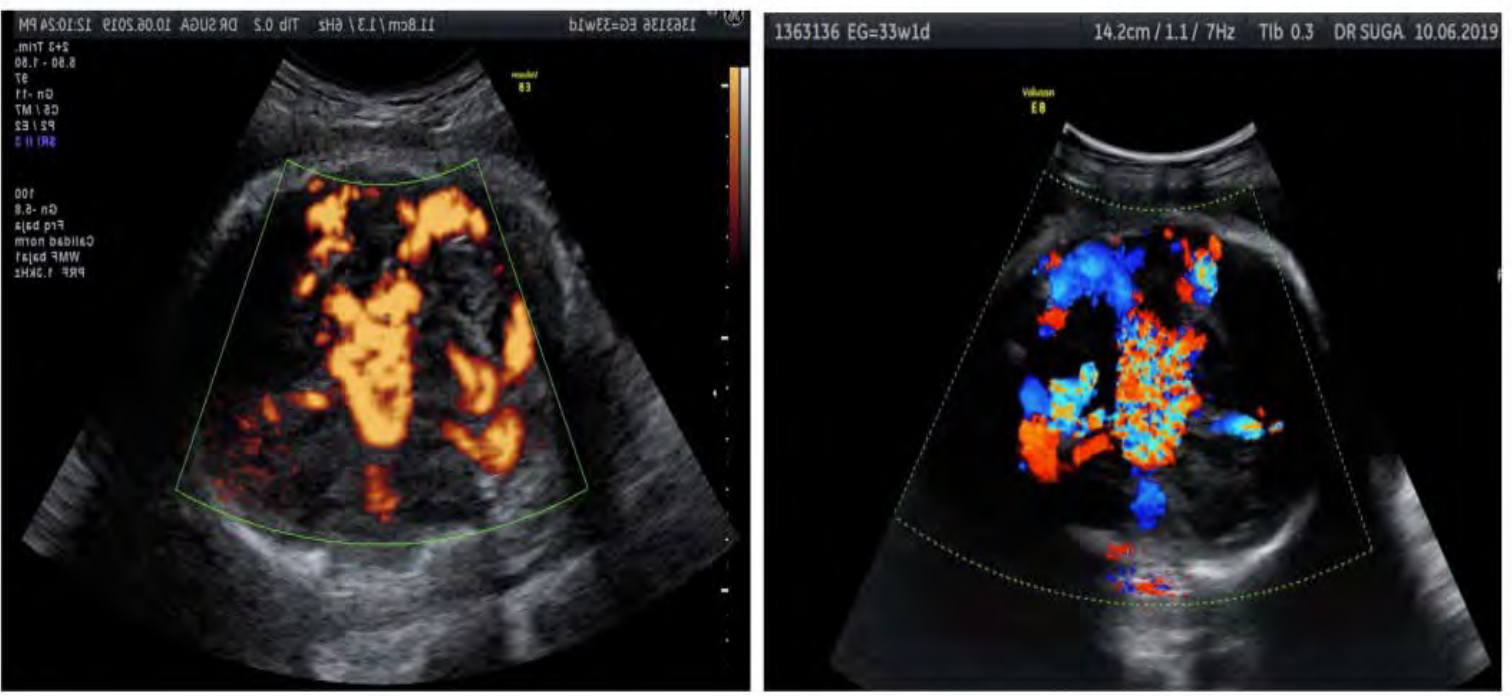

Figura 5 
pupilas). Cabecera $30^{\circ}$. Pase a $\mathrm{UCl}$ y evaluación por Cardiología.

Cardiología lo evalúa y concluye cardiomegalia no asociada a patología estructural cardiaca (Figura 5).

El recién nacido evoluciona desfavorablemente falleciendo al $8^{\circ}$ día de nacido.

\section{DISCUSIÓN}

Desde la publicación de Giancarlo Mari en $2000^{1}$ la evaluación Doppler de la VSMax-ACM se ha convertido en el método de elección para la evaluación no invasiva del riesgo de anemia fetal, no solo por su alto valor predictivo negativo (100\%) sino por su reproducibilidad. A pesar que el mismo autor reconoce una tasa de falsos positivos de $12 \%$, se considera el gold estándar para el tamizaje de anemia fetal. Estudios recientes sugieren, sin embargo, que existen diferencias en los VSMax-ACM entre las diferentes poblaciones ${ }^{2-4}$. Existen en la literatura varios reportes de casos de falsos positivos de anemia fetal severa debidos a patologías fetales como alfa talasemia ${ }^{5,6}$ o cardiomiopatía hipertrófica ${ }^{7}$. Maisonneuve ${ }^{8}$ realiza un estudio de cohorte retrospectiva en 150 gestantes con isoinmunizacion severa en quienes mide el VPS-ACM entre las 34 y 37 semanas observando una tasa de falsos positivos de 34.4\%. En el año 2019 se publico una revisión sistemática con meta-análisis acerca del rendimiento del PVS-ACM en la predicción de anemia fetal la cual concluye que en fetos no transfundidos la sensibilidad es de solo $73 \%$ para una tasa de falsos positivos de $10 \%{ }^{9}$.

Lacunza $^{10}$ reporta el caso de un feto de 37 semanas que presentó un PVS-ACM de $109 \mathrm{~cm} / \mathrm{seg}$ (>1.5 MoM) cuya hemoglobina al nacer fue de $18.1 \mathrm{~g} / \mathrm{dL}$. lo cual sería explicado por el aumento del gasto cardiaco relacionado a la cardiopatía o al aumento del flujo sanguíneo al cerebro en relación al estado hipoxico fetal.

Reportamos un caso de un feto con una patología vascular del Sistema Nervioso Central (aneurisma de $\mathrm{ACM}$ ) que generó una miocardiopatía dilatada secundaria con cardiomegalia y disfunción cardiaca de gasto alto y aumento de la VSmax-ACM por encima de $1.5 \mathrm{MoM}$ (evaluado por 3 examinadores diferentes) y que al nacer no presentó anemia al nacimiento. Cabe señalar que la miocardiopatías hipertróficas detectadas en el periodo fetal pueden ser transitorias ${ }^{11-13}$ debido a la sobrecarga del ventrículo derecho, aumento del gasto cardíaco y circulación hiperdinámica; pero también pueden deberse a enfermedades metabólicas, administración prenatal de corticoides y diabetes materna ${ }^{12}$. El diagnóstico ecocardiográfico se basa en el hallazgo de un engrosamiento del septum interventricular $(4.77 \mathrm{~mm}$ en promedio). La cardiomiopatía hipertrófica regresiona espontáneamente al cabo de 3 ó 6 meses, con cuidados de soporte, restricción de fluidos, diuréticos y oxígeno; sin embargo hay un grupo que pude llegar a requerir cirugía (miotomia septal) siempre y cuando la severidad de la hipertrofia septal y la obstrucción de los tractos de salida causen sintomatología clínica significativa a pesar de la terapia médica.

Las miocardiopatías secundarias presentan indicadores pronósticos ${ }^{12}$ pero en general la evolución del RN depende de la condición primaria como una MAV cerebral en este caso.

En conclusión, todos los fetos con PVS-ACM elevado (> $1.50 \mathrm{MoM}$ ) no necesariamente tienen anemia severa. Se debe tener en cuenta otras causas como alfa talasemia o cardiomiopatía hipertrófica. Debemos sospechar esta última patología en casos de madres diabéticas, fetos en estado fetal no tranquilizador, fetos con malformaciones vasculares cerebrales o fetos que han recibido múltiples dosis de corticoides para maduración pulmonar. Considerar la utilización del Doppler de la arteria cerebral media una herramienta de tamizaje de anemia fetal severa y no diagnóstica, siempre en el contexto del cuadro clínico materno fetal.

Financiamiento: Autofinanciado.

Conflicto de interés: Los autores declaran no presentar ningún conflicto de interés.

\section{REFERENCIAS BIBLIOGRÁFICAS}

1. Mari G. Noninvasive diagnosis by doppler ultrasonography of fetal anemia due to maternal red-cell alloimmunization. $\mathrm{N}$ Engl J Med 2000; 342:9-14.

2. Kumar M, Umrawal T, Singh A. Middle cerebral artery Doppler reference centile charts for the prediction of fetal anemia in a population from India. Int J Gynaecol Obstet 2017; 139: 307-311.

3. Tan KB, Fook-Chong SM, Lee SL, Tan LK. Foetal peak systolic velocity in the middle cerebral artery: an Asian reference range. Singapore Med J 2009; 50: 584-586.

4. Tarzamni MK, Nezami N, Gatreh-Samani F, Vahedinia S, Tarzamni M. Doppler waveform indices of fetal middle cerebral artery in normal 20 to 40 weeks pregnancies. Arch Iran Med 2009; 12: 29-34.

5. Kachewar, S. G., \& Sankaye, S. B. Elevated fetal middle cerebral artery peak systolic velocity: Anemia if not; then what. Int J Anat Radiol Surg, 2014;3(4), 15-18.

6. Maguire, K., Johnson, A., Ou, C. N., Lantin, R. L., \& Moise, K. J. Elevated middle cerebral artery peak systolic velocity without fetal anemia in a case of homozygous athalassemia-1. Prenatal Diagnosis: Published in Affiliation With the International Society for Prenatal Diagnosis, 2008;28(1), 72-74.

7. Heyborne, K. (2009). Elevated Middle Cerebral Artery Peak Systolic Velocity in a Nonanemic Fetus with Alpha-Thalassemia Trait. Obstetrics and gynecology international, 2009.

8. Maisonneuve E, Jayot A, Friszer S, Castaigne V, Cynober E et al. Accuracy of Middle Cerebral Artery Doppler Assessment 
between 34 and 37 Weeks in Fetuses with Red Cell Alloimmunization. Fetal Diagn Ther 2017; 42(3):225-31 DOI: 10.1159/000456661.

9. Martinez-Portilla, R. J., et al. "Performance of fetal middle cerebral artery peak systolic velocity for prediction of anemia in untransfused and transfused fetuses: systematic review and meta-analysis." Ultrasound in Obstetrics \& Gynecology 2019; 54(6):722-731.

10. Lacunza R, Zumaeta I. Cardiomiopatía por cytomegalovirus en el feto. Reporte de un caso. Rev Peru Ginecol Obstet 2019;65(3):367-371 DOI: https://doi.org/10.31403/rpgo. v66i2197.

11. Fetal Cardiomyopathies Pathogenic Mechanisms Hemodynamic Findings, and Clinical Outcome. Circulation 2002 106(5): 585-591.
12. Yunis, KA., Bitar, FF., Hayek, P., Mroueh, SM., \& Mikati, M. Transient hypertrophic cardiomyopathy in the newborn following multiple doses of antenatal corticosteroids. American journal of perinatology, 1999;16(01), 17-21.

13. Vaillant, M. C., Chantepie, A., Casasoprana, A., Chamboux, C., Suc, A. L., Gold, F., \& Laugier, J. Transient hypertrophic cardiomyopathy in neonates after acute fetal distress. Pediatric cardiology, 1997;18(1), 52-56.

\section{Correspondencia:}

Erasmo Huertas Tacchino

Dirección: Jr. Parque Ayacucho 189 Dpto. 302 Pueblo Libre

Correo: erasmohuertas@hotmail.com

Teléfono: 999143288 